\title{
V. SCHLUSSBETRACHTUNG UND AUSBLICK
}

Widerstand durch Planung - Planung als Widerstand: Dies war die kontinuierliche Motivation der deutschen politischen Emigration, sich in den Jahren von 1933 bis 1945 intensiv dem Europagedanken zu widmen. In der politischen und gesellschaftlichen Isolation des Exils entwickelte sich die Idee eines gemeinsamen Europas nicht nur zu einem in vielerlei Hinsicht konkretisierten Denkmodell für die Zukunft des Kontinents nach der Überwindung des nationalsozialistischen Terrors, sondern geriet für viele politische Flüchtlinge auch zum Fokus einer neuen, der Idee der Nation übergeordneten und sie zugleich überwindenden Identität.

Losgelöst von nationaler Regierungsverantwortung und dem Zwang zu tagespolitischer Rücksichtnahme, losgelöst auch vom Druck der öffentlichen Meinung, konnte das deutsche Exil die Europaidee einer rückhaltlosen und zunehmend entdogmatisierten Praktikabilitätsprüfung unterziehen. In der vagen Formulierung einer unverbindlichen Idee war der Europagedanke bei den exilierten Linkssozialisten, Sozialdemokraten, Liberalen, Katholiken, Konservativen und Nationalkonservativen schnell auf breite Zustimmung gestoßen, die anfangs noch in der kaum differenzierten Europabegeisterung der frühen Weimarer Zeit wurzelte. Bezeichnenderweise stellten die mit dem Münchner Abkommen einsetzenden Kontroversen über Detailfragen die generelle Konsensfähigkeit des Europagedankens nicht in Frage, sondern stärkten - trotz vieler konzeptioneller Divergenzen im einzelnen - die einende Kraft des europäischen Ideals. Dabei wurde der Begriff „Europa“, der in den ersten Exiljahren mangels konkreter Europapläne häufig auf seinen geographischen Gehalt reduziert worden war, nun politisch definiert. In dieser politischen Definition war Europa als politisches Zukunftsmodell ebenso enthalten wie als in sich verflochtener Wirtschaftsraum, als historischer Ort, als durch gemeinsame Traditionen verbundener Kulturkreis und nicht zuletzt als kollektiver Erfahrungsraum. Mit der letztgenannten Bedeutung Europas wurde die Schicksalsgemeinschaft des Exils mit den europäischen Widerstandsgruppen und den Bevölkerungen der nationalsozialistisch besetzten Länder angesprochen, die den deutschen Emigranten das Bewußtsein vermittelte, in einen größeren politischen Zusammenhang gestellt zu sein.

Das zentrale Charakteristikum des Europadiskurses im deutschen Exil waren jedoch nicht die divergierenden Definitionen des Begriffs „Europa“, sondern die Konzeptionalisierung des Europagedankens als politischer Lösungsansatz für die vielfältigen, spezifisch europäischen Probleme. In ständiger Reibung mit der Realität des nationalsozialistisch beherrschten Europas und des entstehenden Ost-West-Konflikts offenbarte der Europadiskurs eine zunehmend pragmatische Grundausrichtung. Das nichtkommunistische Exil durchlief einen kontinuierlichen Lernprozeß, der sich im wesentlichen auf 
zwei Ebenen abspielte: Auf der Erkenntnisebene fand eine allmähliche Europäisierung des politischen Denkens statt, die als innere Rückbesinnung auf die europäischen Gemeinsamkeiten umschrieben werden kann. Auf der Handlungsebene war eine von differenzierten Analysen begleitete Verwandlung des nebulösen Schlagwortes „Europa “ in ein konkretes Ordnungskonzept zu beobachten, das im einzelnen unterschiedliche Ausprägungen erhielt. Dabei wurde mehrheitlich die Einordnung des europäischen Staatensystems in einen modifizierten Völkerbund gefordert, so daß die Völkerbundsdiskussion mit dem Europadiskurs untrennbar verbunden war.

Bedingt durch die dramatischen Veränderungen der weltpolitischen Situation, insbesondere die Entstehung des Ost-West-Konflikts, bewegten sich die anfangs weit auseinander liegenden Positionen zur Gestaltung der europäischen Zukunft tendenziell aufeinander zu. Gegen Ende des Zweiten Weltkrieges kann in einigen zentralen Punkten sogar eine von der großen Mehrheit des deutschen Exils getragene Übereinstimmung konstatiert werden. Anders als nach 1918 einte das Exil im Laufe der Jahre die Einsicht, daß nur ein wirtschaftlich und politisch vereinigtes Europa die Fehlentwicklungen der jüngsten Vergangenheit beheben und den vielfältigen Anforderungen der Zukunft mit adäquater Problemlösungskompetenz begegnen könne.

Der Erkenntnisprozeß, der zu dieser Einsicht führte, soll im folgenden anhand der übergeordneten Entwicklungslinien des Europadiskurses kurz skizziert werden. Damit wird zugleich die erkenntnisleitende Frage zusammenfassend beantwortet, bevor die Pläne des Exils - eingeordnet in den historischen Gesamtzusammenhang - auf ihre Tragfähigkeit und ihren Gegenwartsbezug überprüft werden. Eine gewisse Nivellierung der erarbeiteten Divergenzen im Detail ist dabei unumgänglich, um die übergeordneten Entwicklungslinien des Diskurses besser herausstellen zu können.

- Der frühe Europadiskurs der Jahre 1933 bis 1937 vereinte mit Ausnahme der Kommunisten alle Gruppen des deutschen Exils in der gemeinsamen Beschäftigung mit dem Konfliktlösungspotential des Europagedankens, offenbarte das Widerstandspotential des Europabegriffs, förderte das europäische Krisenbewußtsein der deutschen Emigranten und arbeitete in unmittelbarer Anknüpfung an die Europavorstellungen der Weimarer Zeit die Friedenssicherung und die wirtschaftliche Prosperität als zwei wichtige Motive einer europäischen Einigung heraus.

- Das Münchner Abkommen bildete den Auftakt zur Konkretisierungsphase des Europadiskurses, in deren Verlauf der politische Begriff „Europa“ zum Leitbild einer neuen Staatenordnung erkoren und konzeptionalisiert wurde.

- Noch bevor der Zweite Weltkrieg ausbrach, waren die wesentlichen Motive einer europäischen Einigung benannt. Als Alternative zur nationalsozialistischen Außenpolitik sollte ein vereinigtes Europa den unterschiedlich interpretierten Prinzipien der Freiheit und Demokratie zum Durchbruch verhelfen und das offenbar gewordene Unvermögen der Nationalstaaten zur 
Gewährleistung von Frieden, Sicherheit und Wohlstand ausgleichen. Durch die Überwindung des Nationalismus und des verabsolutierten Souveränitätsverständnisses erhoffte sich das politische Exil zugleich die Lösung der deutschen Frage und die friedliche Regelung der Nationalitätenkonflikte in Europa.

- Die Tabuisierung der Ostoption nach dem Hitler-Stalin-Pakt schuf auch im linkssozialistischen Exil das europäische Motiv der antibolschewistischen Abwehr und leitete die Annäherung der sozialistischen und bürgerlichen Europavorstellungen ein.

- Die seit der Niederlage Frankreichs im Juni 1940 evidente Möglichkeit einer außereuropäischen Lösung der europäischen Frage wurde dem Exil mit dem deutschen Angriff auf die Sowjetunion und dem Kriegseintritt der USA zur Gewißheit. Damit begann eine intensive Debatte um die Selbstbehauptung Europas im aufziehenden Ost-West-Konflikt.

- Als Voraussetzung einer europäischen Selbstbehauptung wurde die Schaffung einer europäischen Identität angesehen. Für das sozialistische Spektrum des Exils handelte es sich dabei um eine primär soziale Identität, die der europäische Sozialismus erst noch schaffen werde, während das bürgerliche Exil an die schon vorhandenen kulturellen Gemeinsamkeiten des christlichen Abendlandes anknüpfen wollte.

- Auf die Verlagerung der alliierten Interessen von der europäischen auf die globale Ebene reagierte das Exil unterschiedlich: Während einige Emigranten weiterhin auf eine kraftvolle europäische Eigendynamik hofften, verzichteten andere auf weitere Europapläne und konzentrierten sich von nun an auf die Neuordnung der Welt. Der weitaus größere Teil des Exils akzeptierte den weltpolitischen Bedeutungsverlust Europas und vertraute auf eine am Wohl des Kontinents orientierte, speziell auf Europa zugeschnittene Nachkriegsordnung der Alliierten. Dabei können wiederum drei Akzentuierungen unterschieden werden: eine dem Westen angenäherte Konzeption, ein eher dem Osten verbundenes Modell und die Position einer strikt äquivalenten Ost-West-Kooperation Europas.

- Die Konferenzergebnisse von Moskau und Teheran zerstörten alle Hoffnungen des Exils auf eine progressive Neuordnung Europas und der Welt. Dem Gedanken einer europäischen Föderation erteilten die „Großen Drei“, insbesondere Stalin und Roosevelt, eine Absage. Stattdessen einigten sie sich mit der Anerkennung der sowjetischen Polenpolitik faktisch auf eine Teilung Europas in Einflußsphären. Statt der von vielen Emigranten erhofften Einbindung Deutschlands in ein europäisches Staatensystem sollte Deutschland aufgeteilt werden. Und die Sicherung des Weltfriedens reduzierte sich auf das traditionell machtpolitische Konzept der „Vier Weltpolizisten“.

- Die Auseinandersetzung um die institutionelle Ausgestaltung eines vereinigten Europas wurde nach dem Hitler-Stalin-Pakt zu einem Eckstein des Europadiskurses. Die Debatten ließen bald drei Hauptrichtungen erkennen: die radikal föderalistische Auffassung einer konsequenten Beschneidung des 
nationalen Selbstbestimmungsrechts und der Schaffung supranationaler Organe, die gemäßigte Variante einer bloßen Konföderation unter Verzicht auf eine starke Zentralgewalt und schließlich die im Kern antiföderalistische Position einer Rückkehr zum Europäischen Konzert unter strikter Beibehaltung des nationalen Selbstbestimmungsrechts.

- Die föderalistischen Europapläne bauten durchweg auf dem Gedanken der Supranationalität auf. Die Einsicht in die Schwäche des Völkerbundes führte zu der Forderung nach einer starken Bundesexekutive mit Sanktionsrecht. Ein internationales Schiedsgericht als europäische Judikative wurde weithin für unverzichtbar erachtet. Als bundeseigene Legislative wurde überwiegend ein Zweikammersystem mit einem Völkerparlament und einem Staatenrat favorisiert.

- Je deutlicher die Emigranten die Gefahr einer Teilung Deutschlands erkannten und je mehr sie von der Notwendigkeit europäischer Selbstbehauptung zwischen West und Ost überzeugt waren, desto vehementer traten sie für eine föderative Neuordnung Europas ein. Ende 1943 war die Mehrheit des deutschen Exils überzeugt, daß eine gesamteuropäische Föderation die einzig sinnvolle Alternative zur Teilung Europas sei und nur sie ein Wiedererstarken der weltpolitischen Bedeutung Europas ermöglichen könne.

- In diesem Zusammenhang ist die zwischen 1941 und $1943 \mathrm{zu}$ beobachtende Reaktivierung der Idee einer europäischen Wirtschaftsunion zu sehen, die als geeigneter Einstieg in eine umfassendere supranationale Gestaltung Europas angesehen wurde. Insbesondere die Linkssozialisten präsentierten nun detaillierte Wirtschaftsföderationspläne. Aus sozialistischer Sicht mußte der europäische Integrationsprozeß notwendig mit der wirtschaftlichen Einigung beginnen, um sodann die soziale Einigung zu ermöglichen.

- Nach der Konferenz von Teheran plädierte ein Teil der deutschen Emigration für eine konstruktive Zusammenarbeit der „Großen Drei“ über das Kriegsende hinaus, um auf diese Weise den Frieden in Europa zu garantieren. Andere favorisierten weiterhin das Modell einer eigenständigen Friedensordnung für Europa, entweder unter Führung eines oder mehrerer europäischer Staaten oder als gleichberechtigter Zusammenschluß ohne Führungsmacht.

Vor dem Hintergrund dieser Untersuchungsergebnisse stellt sich nun die Frage nach der Tragfähigkeit der im deutschen Exil entworfenen Europakonzepte: Hatten die deutschen Exilanten die Herausforderungen der Nachkriegszeit erkannt? Boten sie sinnvolle, realisierbare Lösungsmodelle an? Inwieweit nahmen sie Denkprozesse der europäischen Integration nach 1945 voraus? Die Tragfähigkeit der im deutschen Exil entwickelten Europapläne bemißt sich nach dem Grad ihres Problemlösungspotentials, dem mit Blick auf den europäischen Integrationsprozeß nach 1945 nachgegangen werden soll. Die folgende Skizze soll und kann eine noch ausstehende fundierte Untersuchung keineswegs ersetzen. Vielmehr dient sie der historischen Einordnung der Untersuchungsergebnisse. 
Zunächst ist festzustellen, daß die Europavorstellungen des deutschen Exils politisch wirkungslos blieben. Weder die Gründungsväter der europäischen Gemeinschaften noch die deutschen Europapolitiker, sofern ihnen in der nicht souveränen Bundesrepublik überhaupt Bedeutung zukam, griffen auf den Ideenreichtum und die zum Teil scharfsinnigen Analysen der Emigranten zurück. Obwohl sich zahlreiche Emigranten in verantwortlichen Positionen der deutschen Nachkriegspolitik und Nachkriegsgesellschaft wiederfanden, konnten nur wenige von ihnen an ihre eigenen Europaideen aus der Zeit des Exils anknüpfen ${ }^{1}$. Dies lag vor allem an der selbst auferlegten und zugleich aufgezwungenen Zurückhaltung vieler Emigranten hinsichtlich der Erinnerungen an das Exil. Man wollte nicht die eigene Reintegration gefährden ${ }^{2}$. Trotz oder gerade wegen der Ressentiments gegen die "Hitlerfrischler" entschieden sich die Remigranten in der Mehrzahl dafür, nach einer gemeinsamen Identität mit den Daheimgebliebenen zu suchen. Diese gemeinsame Identität war in der kollektiven Aufgabe des Wiederaufbaus Westdeutschlands, in der antibolschewistischen Grundstimmung der westdeutschen Nachkriegsgesellschaft und nicht zuletzt in der gemeinsamen Verdrängung der Erinnerungen an den Nationalsozialismus gefunden. Diese "Kontinuität in schweigender Anpassung " 3 wurde sicherlich durch die pauschale Stigmatisierung der Kollektivschuldthese gefördert.

Zwei grundlegende Faktoren bestimmten die europäische Situation im Jahre 1945: einerseits der Niedergang Europas und die Manifestation der globalen Dichotomie, andererseits die innereuropäische Rückbesinnung als Reaktion auf die sichtbar gewordenen nationalstaatlichen Defizite ${ }^{4}$. Da weder die Europäer noch die Alliierten in der Lage gewesen waren, diese beiden Grundfaktoren rechtzeitig aufeinander abzustimmen, offenbarte sich in der Nachkriegsordnung des geteilten Europas ein ordnungspolitisches Vakuum. Während des zwölfjährigen Exils hatten die deutschen politischen Flüchtlinge diese Problematik erkannt und in vielen Detailstudien analysiert. Im Verlaufe des Europadiskurses arbeiteten sie im wesentlichen vier Problemkreise der europäischen Nachkriegszeit heraus, als deren Ursache sie übereinstimmend das diesbezügliche Versagen der Nationalstaaten benannten: die Ablösung des nationalstaatlichen Ordnungsprinzips in Europa durch

1 Zur politischen Remigration insgesamt vgl. KROHN, ZUR MÜHLEN (Hg.), Rückkehr und Aufbau 1997; FoITZIK, Politische Probleme der Remigration 1991; PAPCKE, Exil und Remigration als öffentliches Ärgernis 1991. Zu den Ausnahmen zählt insbesondere die saarländische Sozialdemokratie, die unmittelbar an Max Brauns Modell des Saarlandes als Ausgangspunkt einer europäischen Föderation anknüpfen konnte. Zu Max Brauns Europavorstellung vgl. PAUL, Max Braun 1988. Zum saarländischen „Emigran-

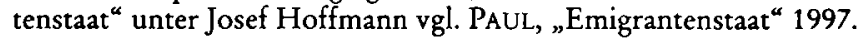

2 Vgl. Rückkehr aus dem Exil und seine Rezeptionsgeschichte. Einleitung, in: KrOHN et al., Handbuch der deutschsprachigen Emigration 1998, Sp. 1157-1160 (1159).

${ }^{3}$ LOTH, Verschweigen und Uberwinden: Versuch einer Bilanz, in: DERS., RUSINEK (Hg.), Verwandlungspolitik 1998, S. 357.

4 So auch LIPGENS, Anfänge 1977, S. 18. 
eine übernationale Friedens- und Sicherheitsordnung, die Schaffung einer europäischen Wirtschaftsföderation als Antwort auf die grenzüberschreitenden Verflechtungen der europäischen Industrie und Wirtschaft, die Einbindung Deutschlands in eine europäische Sicherheitsarchitektur zur Vermeidung künftiger deutscher Aggressionen sowie die Abwendung des weltpolitischen Bedeutungsverlustes für Europa durch eine eigenständige Position im Ost-West-Konflikt. Diese vier europäischen Herausforderungen deckten sich mit den „Antriebskräften“ des europäischen Integrationsprozesses nach $1945^{5}$.

Das deutsche Exil hatte nicht nur die vierfache Problematik der europäischen Nachkriegszeit vorgedacht, sondern auch Lösungsansätze entwickelt, die gegen Ende des Exils ein hohes Problemlösungspotential bis in Detailfragen der Institutionalisierung und Stimmengewichtung hinein erkennen ließen. Dabei handelte es sich überwiegend um umsetzungsfähige Vorschläge, die die analysierten Probleme einer pragmatischen Lösung zuzuführen versprachen.

Die ersten beiden Problemkreise einer neuen Friedens- und Wirtschaftsordnung wollten die meisten politischen Flüchtlinge in den letzten anderthalb Kriegsjahren in einer umfassenden Europaföderation mit einer starken Bundesgewalt aufgelöst sehen. Überwiegend wurde eine Kombination aus wirtschaftlicher und politischer Integration gefordert, zumal ein gemeinsamer Wirtschaftsraum nicht nur den ökonomischen Erfordernissen gerecht würde, sondern auch den innereuropäischen Frieden gewährleisten könne. Waren die Aussichten eines solchen Vorhabens 1945 trüb gewesen, änderte sich dies zwei Jahre später. Nachdem die Sowjetunion eine Beteiligung am Marshall-Plan abgelehnt hatte, war die Teilung Europas in Ost und West evident und kaum mehr zu revidieren. Die USA hatten das wirtschaftliche Potential des europäischen Wiederaufbaus für die amerikanische Wirtschaft erkannt: Hier bot sich die seltene Gelegenheit, sich nicht nur einzelstaatliche Märkte zu erschließen, sondern gleich den gesamten westeuropäischen Wirtschaftsraum für den Absatz US-amerikanischer Produkte zu sichern und auf diese Weise die erhoffte Steigerung des US-Exports herbeizuführen. Folglich fand die Idee einer wirtschaftlichen Föderation Westeuropas nun die Unterstützung Amerikas, und innerhalb von nur elf Monaten reifte der Plan von Monnet und Schuman vom Mai 1950 zum Vertrag über die erste supranationale Wirtschaftsorganisation: die EGKS. 1957 folgte im Rahmen der Römischen Verträge die Gründung der EWG und der EURATOM. Damit war in kürzester Zeit eine beachtliche Integrationsdichte erreicht worden.

Jean Monnets Vorschlag zur Gründung einer europäischen Wirtschaftsföderation in den kriegswichtigen Bereichen Kohle und Stahl war letztlich nicht neu, denn die ihn tragenden Erwägungen waren bis in institutionelle

5 Vgl. Loth, Prozeß der europäischen Integration 2000, S. 18 ff. sowie Ders., Identity and Statehood 2000, S. $25 f$. 
Details von den europäischen Widerstandsgruppen vorgedacht worden. In der deutschen Emigration hatten insbesondere die Wirtschaftskonzeptionen der Sozialisten Kreyssig, Fliess und Monte die Idee einer Montanunion mit einem übernationalen Verwaltungsapparat vorweggenommen. Wie Monnet hatten auch die deutschen Sozialisten die stille Hoffnung auf einen spill-overEffekt gehegt: das Übergreifen der wirtschaftlichen Integration auf den politischen Bereich. Wie die deutschen Sozialisten, so verfolgten auch Jean Monnet und Robert Schuman neben dem ökonomischen Zweck das Ziel einer Frieden sichernden Funktion der EGKS, indem eine erneute deutsche Hegemonie ausgeschlossen werden sollte. Darüber hinaus verband Monnet mit der EGKS die Hoffnung auf eine langfristige Ausweitung zum Kern einer europäischen Regierung ${ }^{6}$. Der Remigrant Kreyssig hatte die EGKS anfangs als kapitalistisch dominierte Organisation abgelehnt, setzte sich dann aber in dem einmal gegebenen Rahmen für eine sozialistische Europapolitik ein 7 . Als Mitglied des Montanparlamentes und später des Europäischen Parlamentes, zu dessen Vizepräsident er avancierte, setzte Kreyssig überzeugend sein im Exil geprägtes Europabewußtsein um.

Anders als die 1957 gegründeten Schwesterorganisationen EWG und EURATOM war Hauptrechtsetzungsorgan der EGKS nicht der intergouvernemental besetzte Rat, sondern die Hohe Behörde als gemeinsame $\mathrm{Be}$ hörde. Diese supranationale Position wurde in Art. 9 EGKSV a. F. ausdrücklich verankert und 'ging mit dem Fusionsvertrag 1965 auf die Kommission über, die nunmehr ein Initiativmonopol inne hat ${ }^{8}$. Herausragendes Kennzeichen der EGKS war somit die Supranationalität, die in Teilen des politischen Exils schon während des Krieges als tragendes Prinzip einer neuen europäischen Ordnung herausgearbeitet worden war. Die Europapläne des deutschen Exils schienen Wirklichkeit geworden zu sein.

Tatsächlich aber sollte sich der europäische Integrationsprozeß schon bald den Einsichten des Exils in zwei zentralen Punkten widersetzen: Erstens

${ }^{6} \mathrm{Zu}$ Monnets weitreichenden Intentionen und Hoffnungen vgl. WILKENS, Jean Monnet, Konrad Adenauer und die deutsche Europapolitik 1999, S. $74 \mathrm{f}$.

7 Vgl. REGIN, Wirtschaft im neuen Europa 1996, S. 76f.

8 THIEMEYER, Supranationalität als Novum 1998, S. 6, irrt in völkerrechtlicher und europarechtlicher Hinsicht, wenn er in seinem ansonsten aufschlußreichen Beitrag behauptet, die "EU“ sei "heute die einzige supranationale Organisation“. Tatsächlich ist nicht die EU, sondern die inkorporierte, aber rechtlich weiterhin eigenständige EG als Zusammenschluß der drei Gründungsorganisationen EWG, EGKS und EURATOM die einzige supranationale Organisation. Die EU dagegen ist eben nicht supranational, denn sie ist nicht identisch mit der EG, sondern setzt sich zusammen aus der EG und den beiden intergouvernemental strukturierten „Säulen" GASP und ZIJ. Darüber hinaus besitzt die EU nach herrschender Meinung noch nicht einmal Völkerrechtssubjektivität, da eine den Art. 281 EGV, Art. 184 EAGV, Art. 6 EGKSV entsprechende Normierung ihrer Rechtspersönlichkeit fehlt (zum Streitstand in der Rechtswissenschaft vgl. STREINZ, Europarecht ${ }^{4} 1999$, Rn. 121 b m. w. N.). Im Gegensatz zur EG ist die EU also keine internationale Organisation im Sinne des Völkerrechts und erst recht keine supranationale. 
wurde das ehrgeizige Projekt einer politischen Integration sehr früh aufgegeben, und zweitens wurde das Prinzip der Supranationalität durch die Rückkehr der beteiligten Staaten zur herkömmlichen Politik des Minimalkonsenses konterkariert.

Die anfängliche Europabegeisterung, die 1949 die Beratende Versammlung des neu gegründeten Europarates dazu veranlaßt hatte, gleich auf ihrer ersten Sitzung mit großer Mehrheit die Einrichtung einer politischen Autorität für Europa zu beschließen - was schließlich am Widerstand Großbritanniens scheiterte -, wich bald nationalstaatlicher Ernüchterung. Bald nachdem mit der Schaffung der supranationalen EGKS die Enge nationalstaatlichen Denkens in beispielloser Weise überwunden worden war, kehrten die europäischen Staaten zur Tradition nationalstaatlicher Interessenpolitik jenseits übergeordneter Ideale zurück. Mehrere Versuche, eine politische Einigung Europas in die Wege zu leiten, scheiterten regelmäßig an nationalen Vorbehalten, und die mißglückten Bemühungen um die Europäische Verteidigungsgemeinschaft (EVG) und die damit verbundene Europäische Politische Gemeinschaft (EPG) im Jahre 1954 bremsten frühzeitig die Dynamik des Integrationsprozesses. Mit der Einheitlichen Europäischen Akte (EEA) von 1986 und dem Vertrag von Maastricht aus dem Jahre 1992 ist das Projekt einer politischen Integration wieder auf die Tagesordnung gesetzt worden. Doch sind die Handlungsspielräume der Gemeinsamen Außen- und Sicherheitspolitik (GASP) und der Zusammenarbeit in den Bereichen Inneres und Justiz (ZIJ) unverändert intergouvernemental angelegt und stehen erst am Anfang ihrer Entwicklung zu effektiven Instrumenten der Gemeinschaft.

Die Supranationalität war als allgemeines Ziel des Einigungsprozesses schon mit dem Scheitern der EVG 1954 in Frage gestellt worden. Bezeichnenderweise fehlte in den Römischen Verträgen zur Gründung der EWG und der EURATOM eine dem Art. 9 EGKSV a. F. vergleichbare Regelung des supranationalen Prinzips. Statt dessen wurde in beiden Organisationen dem Rat als Vertretungskörperschaft der Mitgliedstaaten die Rechtsetzungsbefugnis übertragen. Durch die Relativierung des Mehrheitsprinzips im sogenannten Luxemburger Kompromiß von 1966, der den Mitgliedstaaten in Angelegenheiten von vitalem Staatsinteresse ein Vetorecht einräumte, wurde das Prinzip der Supranationalität für die politische Praxis der Europäischen Gemeinschaften faktisch aufgehoben.

Zum drittgenannten Problemkreis, der Lösung der deutschen Frage im europäischen Kontext, hatte das Exil die Abrüstung und Einbindung Deutschlands in eine gemeinsame Sicherheitsstruktur entwickelt, nachdem man sich von der Vorstellung einer europäischen Schlüsselposition Deutschlands hatte verabschieden müssen. Dieses Konzept, das nicht von allen Emigranten mitgetragen wurde, war als Alternativlösung zu der von den Alliierten anvisierten Zerstückelung Deutschlands entworfen worden. Deutschland wurde dennoch in vier Besatzungszonen geteilt, aus denen 1949 zwei deutsche Staaten hervorgingen, doch wurde zumindest Westdeutschland eng in die west- 
europäische Gemeinschaft eingebunden. Die Idee der EGKS diente nicht zuletzt dem Ziel, das deutsche Wirtschaftspotential in eine gemeinsame Organisation zu integrieren, um seinen Mißbrauch zu verhindern und den europäischen Frieden zu sichern. Nach der deutschen Vereinigung wurde - von den Emigranten selbstverständlich nicht vorauszusehen - mit Erfolg die friedliche und freiwillige Einbindung Gesamtdeutschlands in ein Europa vollzogen, das sich anschickt, wieder zu Gesamteuropa zusammenzuwachsen. Interessanterweise kommt dem vereinigten, souveränen Deutschland innerhalb der Europäischen Union nun in der Tat eine Schlüsselrolle als wirtschaftlicher und - gemeinsam mit Frankreich - politischer Schrittmacher der Integration zu - eine Schlüsselrolle, die das deutsche Exil angesichts der absehbaren deutschen Niederlage lange vor dem Ende des Zweiten Weltkrieges mehrheitlich ad acta gelegt hatte.

Mit dem vierten im Exil analysierten Problemkreis der europäischen Selbstbehauptung zwischen den beiden Weltmächten Sowjetunion und USA hatten die politischen Flüchtlinge die Konzeption der "Dritten Kraft" vorweggenommen, die nach Kriegsende vor allem von der französischen Politik verfolgt wurde. Sicherlich hatten alle Emigranten die frühzeitige Eskalation des Ost-West-Konflikts zum Kalten Krieg sowie die ebenso frühzeitige Zementierung der machtpolitischen Verhältnisse in Europa befürchtet. Das konkrete Ausmaß aber konnten sie kaum vorhersehen. Insbesondere die Reduktion der gesamteuropäischen Integration auf die Integration Westeuropas nur zwei Jahre nach Kriegsende und die damit eng verknüpfte Funktionalisierung des Integrationsprozesses im Systemkonflikt übertraf die meisten Erwartungen. Mit der scheinbar widersprüchlichen Parallelentwicklung der Teilung Europas sowie Deutschlands einerseits und der westeuropäischen Einigung mit der deutsch-französischen Aussöhnung andererseits war eine Situation geschaffen worden, dem das im Exil mehrheitlich verfochtene Konzept einer weltpolitisch eigenständig agierenden Europaföderation nicht mehr gerecht wurde. Denn der westeuropäische Integrationsprozeß stand von Beginn an unter dem Einfluß der USA, die in der 1949 gegründeten Bundesrepublik und dem traditionellen Verbündeten Großbritannien zwei europäische Stützen hatte. Zudem stand Westeuropa wegen der großzügigen Wiederaufbauhilfe in Amerikas moralischer und finanzieller Schuld. Unter diesem Aspekt ist es nicht verwunderlich, daß im Rahmen der Vertragsverhandlungen zu EWG und EURATOM die öffentliche Diskussion kaum zur politischen Funktion der europäischen Organisationen im west-östlichen Spannungsfeld Stellung nahm.

Noch bevor sich Europa gegenüber den USA politisch emanzipieren konnte, zerfiel das Reich der Sowjetunion und mit ihm die Illusion des Staatskommunismus. Damit erübrigte sich eine eigenständige Position Europas im bisherigen Systemkonflikt.

Zusammenfassend läßt sich festhalten, daß das deutsche Exil die vier wesentlichen Problemkreise der europäischen Nachkriegszeit erkannt und 
hierzu tragfähige Lösungskonzepte vorgeschlagen hat. Wenngleich bei den Gründungen der drei europäischen Gemeinschaften in keiner Weise auf die Ideen des deutschen Exils zurückgegriffen wurde, fanden doch viele zentrale Forderungen der Emigranten in der Entwicklung des europäischen Integrationsprozesses nach 1945 ihre Bestätigung. Ohne den vielschichtigen Europadiskurs der Jahre 1933 bis 1945 im deutschen Exil und in den europäischen Widerstandsgruppen ist die Entstehungsgeschichte der europäischen Integration nicht angemessen zu erfassen.

Vor dem Hintergrund dieses Befundes stellt sich die Frage nach der bleibenden Wirkungsmacht des Europadiskurses im Exil: Inwiefern kann die Auseinandersetzung mit den Europakonzeptionen des deutschen Exils heute noch sinnvoll sein? Worin liegt der Gegenwartsbezug dieser Europapläne für den heutigen Stand des Integrationsprozesses?

Für die Beantwortung dieser Frage ist es hilfreich, sich zunächst die verschiedenen Funktionen des Europadiskurses im deutschen politischen Exil zu vergegenwärtigen, die im folgenden stichwortartig aufgezählt seien:

- die Kompensationsfunktion: Kompensation für verlorene Illusionen, insbesondere die Vorbildfunktion der Sowjetunion als sozialistischer Vorzeigestaat, das linksrevolutionäre Potential der deutschen Arbeiterbewegung, die Schlüsselrolle Deutschlands für Europa, Deutschland als abendländische Kulturnation sowie die durch Hitlers übersteigerten Nationalismus diskreditierte Idee der Nation als ordnungspolitische Grundkategorie in Europa;

- die Abgrenzungsfunktion des „Anderen Deutschlands“ gegenüber dem Nationalsozialismus - Abgrenzung nach außen;

- die Legitimationsfunktion für das Exil als Teil des Widerstandes: der Europadiskurs als Plattform der Widerstandsarbeit, der politischen Gegenentwürfe des „Anderen Deutschlands“ - Legitimation nach innen, gegenüber dem eigenen Volk;

- die Individualisierungsfunktion durch gruppenübergreifende Diskussionen, Parteiaustritte und -übertritte sowie neue, zunehmend die Parteilinien ignorierende Gruppengründungen: Auflösung der parteiideologischen Landschaft von Weimar mit der Folge, daß der unabhängig denkende Europäer in den Vordergrund trat;

- die Solidarisierungsfunktion als logische Ergänzung der Individualisierungsfunktion: Der Europadiskurs als Ausdruck eines begrenzten Kollektivbewußtseins des deutschen politischen Exils, verbunden in der gemeinsam erlebten Europäisierung und der Forderung nach Überwindung des Nationalismus. Trotz aller Divergenzen verursachte die gemeinsame Schicksalserfahrung des Exils doch eine gewisse Annäherung des politischen Denkens, insbesondere hinsichtlich des westlichen Demokratieverständnisses, des Antifaschismus und des politischen Selbstverständnisses als Alternative zu Hitler';

${ }^{9}$ Vgl. insoweit auch die Selbsteinschätzung des Emigranten LOEWY, Literarische und politische Texte, Bd. 2, 1982, S. 16. 
- die Stabilisierungsfunktion: Stabilisierung des Exils als Ort politischer Kultur, demokratischer Übung und freiheitlichen Meinungsaustauschs, mithin als Ort kultureller Selbstvergewisserung angesichts der Barbarisierung Europas durch den Nationalsozialismus;

- die Profilierungsfunktion: Politische Profilschärfung im Exil, insbesondere in der Föderalismusdebatte. Dies bedeutete zugleich die Chance, sich auch in der Heimatlosigkeit als politischer Mensch begreifen zu können.

Diese sieben Funktionen des Europadiskurses verbindet vor allem eines: Sie veranschaulichen die durch die gesellschaftliche und politische Isolation des Exils zwangsläufig hervorgerufene Selbstbezogenheit der deutschen Emigranten. Alle sieben Funktionen unterstreichen letztlich die paradox erscheinende, exiltypische Entwicklung: Die Auseinandersetzung um ein neues Deutschland in einem neuen Europa, die gerade in dem Gefühl deutscher und europäischer Interessensolidarität geführt wurde, warf die Emigranten auf sich selbst zurück, überließ sie dem mehr oder weniger abgekapselten Erfahrungsraum Exil, der weder zum innerdeutschen Widerstand noch zu den Regierungen der kriegführenden Länder Kontakte aufrecht zu erhalten vermochte und der politisch folgenlos blieb.

Umso mehr bot das Leitbild Europa den Emigranten eine neue, positiv besetzte Identität als antifaschistische Europäer. Nach anfänglichem Zögern und Festhalten an der deutschen Nation als Identifikationsobjekt mußten die Emigranten 1938 erkennen, daß sie sich auf eine längere Zeit des Exils und der Heimatlosigkeit einrichten mußten. Die Identifizierung mit Deutschland fiel zunehmend schwerer, und auch die Parteien fielen nach und nach als identitätsstiftende Bezugsobjekte aus. Viele Linkssozialisten hatten anfangs nicht die Nation, sondern den internationalen, sowjetfreundlichen Sozialismus als geistige Heimat angesehen. Doch wurde diese Illusion mit dem Hitler-Stalin-Pakt zerstört. Mit dem Ausfall der Sowjetunion einerseits und der deutschen Nation andererseits begann für die sozialistischen ebenso wie für die bürgerlichen Emigranten die Suche nach einer neuen Identität. Diese Suche führte viele Emigranten über die negative Identifizierung mit allen NSGegnern zur positiven Identifizierung mit dem Leitbild eines vom Faschismus befreiten, vereinigten Europas. Mithin entwickelte das Exil wichtige Ansätze einer Verdichtung des seit 1918 virulenten, aber bisher nebulösen Europabewußtseins zu einer europäischen Kollektividentität.

An dieser Stelle wird die Bedeutung der Exildiskussionen für den heutigen Prozeß der Europäisierung deutlich. Denn der Lernprozeß, der dem deutschen Exil schließlich die Einsicht brachte, daß ein wie auch immer geartetes vereinigtes Europa seinen Völkern eine europäische Identität bieten müsse, um sich als politischer Ordnungsrahmen langfristig durchsetzen zu könnendiesen Lernprozeß hat die deutsche Nachkriegsgesellschaft bis heute nicht ernsthaft aufgenommen. Die Suche nach einer gemeinsamen europäischen Identität hat gerade erst begonnen. Sie verläuft bis dato hektisch, aber orientierungslos. In dieser Situation kann die Vielfalt des Europadiskurses im Exil 
dem heutigen Integrationsprozeß hilfreiche Anhaltspunkte für die gezielte Entwicklung einer europäischen Identität bieten ${ }^{10}$.

Diese erste Erkenntnis bedarf der weiteren Konkretisierung, die nun in einem gedanklichen Dreischritt vollzogen werden soll. Zunächst muß in der gebotenen Kürze geklärt werden, ob eine europäische Identität aus heutiger Sicht überhaupt erforderlich ist. Anschließend ist zu fragen, auf welche Weise eine solche Kollektividentität, wenn man sie denn für erforderlich hält, geschaffen werden kann. Erst dann können auf der Basis der Ergebnisse dieser Arbeit die konkreten Ansatzpunkte benannt werden, die sich für einen gedanklichen Rückgriff auf den Europadiskurs des deutschen Exils anbieten.

Der Soziologe Karl W. Deutsch konstatierte 1972 nicht zu Unrecht, daß sich die Wahrnehmung der breiten Bevölkerungsschichten Europas „nur sehr langsam auf die Vorstellung einer europäischen Einheit" zubewege ${ }^{11}$. Zwischenzeitlich hat der Integrationsprozeß mit der intensivierten Debatte um eine europäische Wirtschafts- und Währungsunion das eher unbestimmte Bewußtsein einer Zusammengehörigkeit der europäischen Völker tradiert, in Teilen möglicherweise sogar vertieft, aber nicht zu einer europäischen Identität verdichtet. Warum nicht?

Eine Identität definiert sich als Bewußtseinskategorie und emotionale $\mathrm{Ka}$ tegorie zugleich. Als Kategorie des Bewußtseins kann sich eine kollektive Identität entweder vergangenheitsbezogen auf gemeinsame Leistungen beziehen oder zukunftsgerichtet auf das einende Gefühl einer gemeinsamen Sendung ${ }^{12}$. Für eine europäische Identität kann insoweit auf die abendländischen Traditionen, die gemeinsame Geschichte und ein aus Christentum und Aufklärung komponiertes Wertesystem verwiesen werden. Auf der emotionalen Ebene jedoch ist das Empfinden der Europäer als „Wir-Gruppe“ im Sinne eines starken, positiv konnotierten Zusammengehörigkeitsgefühls erforderlich. Ohne diese Gefühlskomponente ist die Entwicklung einer europäischen Identität nicht denkbar. Ernest Renans ebenso berühmter wie treffender Ausspruch über die Nation ist auch auf „Europa" anwendbar: Europa ist ein tägliches Plebiszit, dessen Fundament nur solange trägt, wie die Menschen sich mehrheitlich mit der konkreten Verfaßtheit Europas identifizieren

10 Für die folgenden Ausführungen wurde auf Einzelnachweise weitgehend verzichtet. Die Gedankengänge sind unter Zuhilfenahme folgender Werke entwickelt worden: KAELBLE, L'Europe „vécue“ 1994; KrRSCH, Nationalstaat im Spannungsfeld 1997; LADEMACHER, Kultur - Region - Nation 2001; LOTH, Identity and Statehood 2000; Ders., Prozeß der europäischen Integration 2000; Ders., Die Deutschen und das Projekt der europäischen Einigung 1992; DERS., Weg nach Europa ${ }^{31996 ; ~ M U ̈ N C H, ~ P r o j e k t ~}$ Europa 1993; THIEMEYER, Supranationalität als Novum 1998.

11 DEUTSCH, Nationalismus 1972, S. 124.

12 Vgl. SCHNEIDER, Identität und Identitätswandel 1998, S. 249. DEUTSCH, Nationalismus 1972, S. $106 \mathrm{f}$. überträgt diese Erkenntnis auf jede Art von Föderation: „Für eine Föderation ist entweder Erfahrung in der Vergangenheit oder eine Erwartung in der Zukunft und etwas Erfahrung im wirtschaftlichen Wachstum, zum mindesten in einigen Einheiten nötig.“ 
können und wollen. Europa muß als Zugehörigkeitsstruktur gelebt werden, um als Idee in der Praxis zu bestehen ${ }^{13}$.

Der Identifizierung breiter Bevölkerungsschichten mit dem europäischen Integrationsprozeß steht bis heute dessen technokratische Handhabung entgegen ${ }^{14}$. Dies gilt selbst für den Vertrag von Maastricht, der in Frankreich mit denkbar knapper Mehrheit das Plebiszit überstand - ein Balanceakt zwischen raison d'État und öffentlicher Meinung. In Deutschland wollte man das epochale Vertragswerk gar nicht erst der Gefahr eines ablehnenden Volksvotums aussetzen und scheute selbst eine unverbindliche Volksbefragung. Mit der Einführung des europäischen Binnenmarktes und dem Euro als gemeinsamer Währung sind die technokratischen Arbeitsziele erreicht worden, ohne daß ein nennenswertes kollektives Zusammengehörigkeitsgefühl damit verbunden gewesen wäre. Einer identitätsstiftenden Wirkung des europäischen Integrationsprozesses und der europäischen Organe fehlte bisher das dahinterstehende übergeordnete Ideal, das der (west)europäischen Bevölkerung als erstrebenswertes Integrationsziel hätte vermittelt werden können.

Die technokratischen Zielsetzungen der EG eignen sich kaum als völkerverbindende Ideale. Als Garant der Friedenssicherung sind bisher die USA angesehen worden. Die Schaffung eines gemeinsamen Wirtschaftsraumes war eine pragmatische Notwendigkeit, aber als völkerverbindendes Ideal ungeeignet. Auch das Ziel der europäischen Eigenständigkeit zwischen Ost und West war, soweit es überhaupt ernsthaft verfolgt wurde, für eine kollektive Vision zu abstrakt und zu wenig emotionsbehaftet. Der Föderativgedanke als Ordnungsprinzip kann ebensowenig überzeugen, da er nicht Selbstzweck ist, sondern nur Mittel zum Zweck sein kann und als Bezugsobjekt eines WirGefühls ebenfalls zu abstrakt ist.

Bis heute wird der Integrationsprozeß nahezu ausschließlich vom rationalen, häufig nationalstaatlich-machtpolitischen Kalkül bestimmt, ohne in den breiten Bevölkerungsschichten emotional verankert zu sein. Nicht einmal die privaten Europa-Initiativen und erst recht nicht die europäischen Intellektuellen haben es vermocht, das Handlungsmonopol der Regierungen bei der Realisierung der Europaidee zu brechen. Dies, obwohl die Europäisierung mit der Übertragung nationaler Teilsouveränität erheblich weiter fortgeschritten ist als je ein anderer Integrationsprozeß. Insoweit ist die begriffliche Differenzierung Guy Kirschs zwischen der durch die Teilübertragung der

${ }^{13}$ Dies betonen die neuesten Studien zur Bewußtseinsgeschichte des europäischen Integrationsprozesses, vgl. stellvertretend KAELBLE, L'Europe „vécue“ 1994, S. 27: "L'émergence de la conscience européenne ne suit pas fidèlement et automatiquement l'évolution d'une société européenne ,vécue par les Européens, c'est-à-dire comprise par eux; elle dépend aussi d'autres facteurs comme les idéaux européens, les institutions européennes, les concepts politiques sur l'Europe, en somme l'Europe, voulue‘." Auch LADEMACHER, Kultur - Region - Nation 2001, S. 352 konstatiert eine Konkurrenz zwischen dem Nationalgefühl und dem „neuen, zunächst noch geforderten, aber noch nicht gelebten Gefühl der europäischen Gemeinschaftlichkeit“.

14 Übereinstimmend LOTH, Identity and Statehood 2000, S. 27. 
Nationalsouveränität erreichten Suprastaatlichkeit und ihrer Ergänzung durch eine noch ausstehende Supranationalität als kollektives Zusammengehörigkeitsgefühl der Europäer bemerkenswert: „Ehe denn der einzelne weiß, $\mathrm{da}$ er ein Deutscher, ein Franzose, ein Italiener oder ein Däne ist, füblt er, $\mathrm{daß}$ dem so ist [Hervorhebungen des Verf., B. S.]." 15

Die Gefahr einer bloßen Suprastaatlichkeit ohne korrelierende Supranationalität liegt primär in den Renationalisierungstendenzen der Mitgliedstaaten. Gerade die Suche nach Identifikationsbezügen kann solchen Renationalisierungstendenzen Vorschub leisten. Denn emotional bindende Identitäten können in den kleineren, besser überschaubaren und aus eigener Lebenspraxis vertrauten Erfahrungsräumen der Nation und der Region eher begründet werden als auf der noch nicht recht faßbaren europäischen Ebene. Für die Glaubwürdigkeit des weiteren Integrationsprozesses und seine Akzeptanz bei den allmählich sensibilisierten Bevölkerungsmassen wird nicht das „Europe pensée“ elitärer Diskurse, sondern vor allem die Erfahrung eines „Europe vécue“ 16 entscheidend sein. Somit ist die Verdichtung des zweifellos vorhandenen Europabewußtseins zu einer kollektiven Identität der Europäer sowohl für den langfristigen Erfolg des bisher erreichten Integrationsgrades als auch für die Legitimation der fortgesetzten Europäisierung unverzichtbar.

Bejaht man die Notwendigkeit einer europäischen Identität, schließt sich die Frage an, auf welche Weise eine solche Kollektividentität geschaffen werden kann. Ein europäisches Zusammengehörigkeitsgefühl setzt eine möglichst enge Verbindung des Erfahrungsraumes Europa mit dem Alltagsleben der Bürger voraus. Dabei muß klar sein, daß eine europäische Identität die nationalen Identitäten vorerst nur ergänzen, nicht aber ersetzen kann. Denn abgesehen von der momentan deutlich stärkeren Bindungskraft der Nation gegenüber Europa ist die nationale Vielfalt Europas, das unterschiedliche Nationalverständnis und die divergierenden Lebensrhythmen selbst Teil der europäischen Kultur - Ausdruck der so oft beschworenen discordia concors. Die fortschreitende Institutionalisierung Europas, die zunehmende europäische Regelungsdichte sowie der gemeinsame Binnenmarkt werden den Europagedanken sicherlich besser vermitteln können als die bloße Symbolik einer Europaflagge oder einer Europahymne. Doch so wichtig diese Entwicklungen für die langfristige Stärkung des Europabewußtseins sind, können sie doch nur identitätsfördernd, nicht aber identitätsstiftend wirken. Hierzu bedarf es einer über den organisatorischen Alltag hinausgehenden Vision, eines

15 KIRSCH, Nationalstaat im Spannungsfeld 1997, S. 11. Kirschs begriffliche Differenzierung ist bislang weder von der Rechtswissenschaft noch von der Politikwissenschaft aufgegriffen worden. Dies ist umso bedauerlicher als auch die Begriffe „international“, "transnational", ja sogar "national" von Grund auf neu überdacht und im Einzelfall auf ihre rationale und emotionale Lebenswirklichkeit überprüft werden müßten.

16 Vgl. KAELBLE, L’Europe „vécue“ 1994. 
europäischen Ideals, zu dem die Europäer eine emotionale Bindung aufbauen können.

In der momentanen Diskussion werden im wesentlichen drei konzeptionelle Ansätze vertreten, auf welche Weise eine europäische Identität geschaffen werden kann: der währungspolitische Ansatz, der auf die identitätsstiftende Wirkung der gemeinsamen Währung Euro abstellt, das regionalpolitische Konzept des Europas der Regionen und der soziokulturelle Ansatz einer Identität der politischen Kultur mit dem Fernziel einer europäischen Gesellschaft.

Seit dem 1. Januar 2002 wird in dreizehn Ländern der Europäischen Union mit derselben Währung bezahlt. Fraglos wird der Umgang mit der gemeinsamen Währung zu einer wichtigen kollektiven Alltagserfahrung werden und damit der notwendigen Vertiefung des Europabewußtseins förderlich sein. Als Bezugsobjekt einer tiefgreifenden emotionalen Bindung aber wird die Währungsunion möglicherweise nicht ausreichen. Zur Begründung muß zunächst auf das fehlende Vertrauen der Märkte in den Euro und dessen lange Zeit schwache Position gegenüber dem US-Dollar verwiesen werden. Darüber hinaus muß der Tatsache Rechnung getragen werden, daß dem Euro anders als der Deutschen Mark - das identitätsstiftende Kollektiverlebnis eines als Gemeinschaftsleistung verstandenen „Wirtschaftswunders“ wohl nicht zuteil werden wird. Somit erscheint der Euro als Fixpunkt einer europäischen Identität kaum prädestiniert.

Das Konzept eines Europas der Regionen, das eng mit dem Subsidiaritätsprinzip verbunden ist, bemüht sich um die Initiierung eines europäischen Identifikationsprozesses mittels einer Stärkung der regionalen Bande. Mit der Reaktivierung und Intensivierung der regionalen Bindungen hofft man, zugleich die Zugehörigkeit der Region zu Europa in Erinnerung zu rufen ${ }^{17}$. Für diesen zweigleisigen Identifikationsversuch spricht, daß er die europäische Identität nicht absolut setzt, sondern den Menschen ihre Bindungen vor Ort läßt, diese sogar unterstützt. Der leichter faßbare und bereits emotional gebundene Erfahrungsraum Region soll zugleich zum Vermittler einer europäischen Zugehörigkeit werden. In der politischen Praxis aber sind die Regionen letztlich von machtpolitischem Eigeninteresse geleitet. Dadurch wird das Konzept des Europas der Regionen unter Vernachlässigung der europäischen Identifikationsebene umgesetzt und verkommt oft genug zu einer einseitigen Relokalisierung der Interessen. Solange sich die Regionen aber nicht als primäre, zumindest gleichwertige Multiplikatoren der europäischen Einheit begreifen, ist die Vorgehensweise, über den regionalpolitischen Umweg eine europäische Identität zu schaffen, schon im Ansatz fruchtlos. Zudem ist der Begriff der Region in Europa mit völlig unterschiedlichen Inhalten erfüllt und erfaßt etwa das Baskenland, Korsika und Schottland ebenso wie die

17 Zum Konzept eines Europas der Regionen vgl. stellvertretend für die umfangreiche Literatur: ClOSTERMEYER, Europa der Regionen 1997. 
deutschen Bundesländer. Vor dem Hintergrund seiner europafernen Handhabung ist das Konzept des Europas der Regionen momentan kaum geeignet, eine europäische Identität zu schaffen, sondern führt $\mathrm{zu}$ einer jedenfalls verzögernden Umleitung des Identifikationsprozesses über die Regionen.

Erfolgversprechender ist der soziokulturelle Ansatz. Nicht die Nivellierung kultureller Besonderheiten, sondern die Betonung des gemeinsamen Kulturverständnisses auf der Basis eines gemeinsamen Wertekataloges ist am ehesten als Grundlage einer europäischen Identität geeignet. Angestrebt wird also eine europäische Identität als Wissen und Wollen der Zugehörigkeit zu einer gemeinsam definierten Wertegemeinschaft, die geeignet ist, beides zu sein: europäisches Ideal und erlebte Kollektiverfahrung.

Für das soziokulturelle Verständnis der europäischen Identität spricht, daß eine Identitätsbildung immer ein kultureller Prozeß sein muß, weil sie gerade über die bloße ratio einer wirtschaftlich oder politisch vernünftigen Integration hinausgeht. Darüber hinaus wäre eine integrative Kulturidentität die logische Fortsetzung des bisherigen Integrationsprozesses: wie die Wirtschaftsintegration zugleich den Keim der - lange hinausgezögerten - politischen Integration war, so trägt die mit GASP und ZIJ vorsichtig begonnene Institutionalisierung einer politischen Integration den Keim einer Kulturintegration in sich. Denn Kultur meint auch politische Kultur, beinhaltet das Bekenntnis zum Verfassungsstaat und zu den demokratischen Umgangsformen. Dazu gehört auch, daß die kulturelle Identität Europas nicht als Abgrenzungsmerkmal gegenüber den nicht zu leugnenden außereuropäischen Einflüssen, also im Sinne einer „Restüberlegenheit der europäischen Kultur" 18 verstanden werden darf, sondern die Einbeziehung und Reflexion der externen Einflüsse zur gegenseitigen Bereicherung anzustreben ist.

Auch sind mit der deutschen Vereinigung und dem Ende des Kalten Krieges gewichtige Gegenargumente entfallen, die zuvor gegen eine kulturelle Identität der Europäer in Stellung gebracht worden waren. Bis zur Vereinigung der beiden deutschen Staaten erschwerte in Deutschland das Fehlen einer nationalen Identität die Suche nach einer übergeordneten europäischen Identität. Die Ersatzidentität der Deutschen Mark konnte dieses gravierende Manko nicht ausgleichen. Dies galt insbesondere gegenüber Großbritannien und Frankreich, die beide über ein ungebrochenes, über Jahrhunderte aufgebautes Nationalbewußtsein verfügen. Dieses Dilemma wurde durch die deutsche Vereinigung 1989/90 nicht ohne weiteres gelöst. Denn einerseits fiel ein Teil der Politik und der Bevölkerung vorübergehend in nationalistische Reflexe zurück, die eine betonte Rückbesinnung auf die nationale Identität forderten und den Blick auf die europäische Idee verstellten. Andererseits können nun auch die Deutschen souveräner mit der Forderung nach einer europäischen Identität umgehen, da ihnen der organische Zwischenschritt der nationalen Identitätsebene wiedergegeben ist.

18 KAELBLE, Europäer über Europa 2001, S. 147. 
Nach dem formalen Ende der europäischen Ost-West-Teilung sind auch die Vorbehalte gegen eine allzu intensive westeuropäische Einigung ohne Einbeziehung der mittel- und osteuropäischen Staaten entfallen. Zugleich hat die dramatische Verringerung der amerikanischen Friedenspräsenzstärke in Europa einer spezifisch europäischen Identitätssuche den Weg geebnet. Eine vorsichtige Emanzipation von den USA in wirtschaftlicher, politischer und gesellschaftlicher Hinsicht erscheint angebracht, ohne sich in eine nur negativ identitätsstiftende Isolation begeben zu müssen.

Anknüpfungspunkt einer europäischen Kulturidentität müßte ein gemeinsames Werteverständnis sein. Hier bietet sich der Wertekanon der Europäischen Menschenrechtskonvention (EMRK) an, ergänzt um die Grundfreiheiten des Maastricht-Vertrages, ein dem Art. 49 EUV vergleichbares Bekenntnis zu Demokratie, Freiheit und Rechtsstaatlichkeit sowie die verbindliche Anerkennung des Föderalismusprinzips und der Europäischen Sozialcharta. Eine andere Grundlage als diejenige eines als Rechtsanspruch und politisches Bekenntnis artikulierten Wertekataloges ist nicht recht ersichtlich. Das vormals identitätsstiftende Christentum ist heute nicht mehr in der Lage, breiteren Bevölkerungsschichten eine politische Idee gefühlsmäßig nahe zu bringen. Auch ein Appell an die einigende Ausstrahlung der abstrakten "Vernunft" kommt nicht in Betracht, denn die Vision einer europäischen république des lettres richtete sich ausschließlich an bestimmte Eliten. Breiteren Bevölkerungsschichten aber wäre dieser Ansatz auch heute nicht vermittelbar.

Für die Entwicklung einer soziokulturellen Identität kann - eher als dem Verwaltungsorgan Kommission - dem Europäischen Gerichtshof (EuGH) eine wichtige Rolle zukommen. Der EuGH nimmt im Integrationsprozeß eine zentrale Funktion ein: Er garantiert die Rechts"staatlichkeit" auf europäischer Ebene, seine Rechtsprechung geht selbst der Rechtsprechung der nationalen Verfassungsgerichte vor. Er überwacht als Judikative die Einhaltung der Verträge, schlichtet Streitigkeiten zwischen Mitgliedstaaten und öffnet seine Säle auch dem einzelnen EU-Bürger. Tatsächlich hat der EuGH über die Auslegung des europäischen Primärrechts hinaus das Gemeinschaftsrecht zu einer Rechtsordnung sui generis entwickelt. Zu beachten ist auch, daß mit der wachsenden Integrationsdichte die Hürden der Klagebefugnis vor dem EuGH verringert und damit die Rechtsprechungskompetenz des obersten europäischen Gerichts weiter ausgedehnt werden wird. Institutionelle Abgrenzungsschwierigkeiten, die sich für die Auslegung der EMRK möglicherweise aus der insoweit kongruenten Prüfungskompetenz des Europäischen Gerichtshofes für Menschenrechte ergeben könnten, wird die politische Praxis voraussichtlich zugunsten des deutlich einflußreicheren EuGH entscheiden. Dafür spricht auch die divergierende politische Bedeutung der beiden dahinterstehenden Organisationen, der EU einerseits und dem schon frühzeitig entpolitisierten Europarat andererseits. Mithin ist der EuGH ein geeignetes Instrument, die politische Kultur Europas zu festigen und der Entwick- 
lung eines europäischen Zusammengehörigkeitsgefühls förderlich zu sein. Vor dem Hintergrund dieser Überlegungen ist es als Integrationsrückschlag zu werten, daß in Nizza die Verabschiedung einer verbindlichen Europäischen Charta der Menschenrechte verschoben wurde und bis dato lediglich ein dürftiger Verweis auf die EMRK in Art. 6 EUV enthalten ist.

Nachdem der soziokulturelle Ansatz einer Identität der politischen Kultur mit dem Fernziel einer europäischen Gesellschaft als erfolgversprechendster Identifikationsversuch vorgestellt worden ist, kann nun die Frage aufgegriffen werden, inwieweit die Auseinandersetzung mit dem Europadiskurs des deutschen Exils bei der Schaffung einer kulturellen Kollektividentität hilfreich sein kann.

Der im Detail äußerst kontroverse Europadiskurs im deutschen Exil kann für die heutige Europadiskussion eine Interpretations- und Orientierungshilfe leisten und dazu beitragen, den Kerngedanken des europäischen Einigungsprozesses offenzulegen: Völkerverständigung und kollektive Friedenssicherung. Dies gilt insbesondere angesichts der heute häufig anzutreffenden Skepsis gegenüber einem mittlerweile institutionalisierten Europa, dessen Rechtsprechung und Verwaltung wachsenden Einfluß auf das Alltagsleben des einzelnen Bürgers ausüben. Die Reaktionen der europäischen Politik auf die Unsicherheit der Bürger weisen häufig Renationalisierungstendenzen auf, und auch der nunmehr unmittelbar betroffene Bürger fragt nach Sinn und Ziel der europäischen Integration, die der bloßen Ideenkiste entronnen und den Kinderschuhen entwachsen ist.

Damit ist die Frage nach der Legitimität des Integrationsprozesses aufgeworfen: Die Einsicht des Exils in die funktionalen Unzulänglichkeiten der Nationalstaaten, die mit den Antriebskräften des Integrationsprozesses nach 1945 übereinstimmten, bilden noch heute die Basis der fortschreitenden Integration. Deshalb ist die Rückbesinnung auf die Vorgeschichte des Einigungsprozesses unerläßlich, um die Legitimation der weiteren Integration auch mit den bisherigen Integrationserfolgen und den sie tragenden Grunderkenntnissen zu intensivieren. Diese Grunderkenntnisse konnten im Exil vorbehaltloser formuliert werden, so daß der Europadiskurs des Exils aufschlußreiche Einsichten für das heutige Integrationsverständnis zu vermitteln vermag.

Darüber hinaus kann die innereuropäische Rückbesinnung des Exils als europäischer Lernprozeß für die heutige Definition einer Identität der politischen Kultur von Bedeutung sein. Auf der Suche nach gemeinsamen europäischen Traditionen, Werten und Interessen konnten sich die deutschen Emigranten im großen und ganzen auf grundlegende Prinzipien der politischen Kultur verständigen: Demokratie, Freiheit, Föderalismus und die Verbindlichkeit der Menschenrechte als Ausdruck der Herrschaft des Rechts. Diese Prinzipien entsprechen dem gemeinsamen Wertekanon, der zu Beginn des 21. Jahrhunderts als Fixpunkt einer soziokulturellen Identität der Europäer anzusehen ist. 
Die Auseinandersetzung mit den eingehend erörterten europäischen Beweggründen und Überlegungen des Exils als Teil der europäischen Geschichte ist geeignet, das individuelle Europabewußtsein zu schärfen und ermöglicht die historische Verortung der Anfänge des europäischen Integrationsprozesses.

Auch die ausführlichen Überlegungen des Exils zur europäischen Eigenständigkeit können sich als bedeutungsvoll für die Schaffung einer Identität der politischen Kultur nach dem Ende des Kalten Krieges erweisen. Im Vordergrund steht heute die kulturelle Selbstbehauptung Europas nach außen und die Homogenisierung der Einheit in Vielfalt nach innen. Nach außen wird sich Europa seiner kulturellen Selbstbehauptung zwischen dem amerikanischen, dem arabischen und dem asiatischen Kulturkreis widmen müssen. Nach innen sieht sich das vereinigte Europa nach der Auflösung der weltpolitischen Machtblöcke der schwierigen Aufgabe ausgesetzt, erstmals eigene Ordnungsfunktionen auf dem europäischen Kontinent auszuüben. Mit Blick auf die anstehende Osterweiterung der Europäischen Union wird sich diese Aufgabe als eine wesentlich kulturelle Herausforderung erweisen, die die Entwicklung unterschiedlicher politischer Kulturen diesseits und jenseits des Eisernen Vorhangs wird homogenisieren müssen, ohne der entstehenden europäischen Gesellschaft die nationalen und regionalen Besonderheiten vorzuenthalten.

Schließlich sind die Europakonzeptionen des Exils fraglos ein bedeutender Teil des Fundaments für die weitergehende Europaforschung, zumal sich die Beiträge des deutschen Exils häufig durch hohen Sachverstand und scharfsichtige Analysen auszeichneten. Es ist zu hoffen, daß durch die Beschäftigung mit dem Europadiskurs der Jahre 1933 bis 1945 der europäische Enthusiasmus, die Begeisterung für eine politische Idee auch in unsere Zeit hinüberwirkt. So könnte der heutige Integrationsprozeß von einer erfolgreichen, von der wirtschaftlichen Vernunft diktierten Vergangenheit in eine ebenso erfolgreiche, von einer soziokulturellen Identität der Europäer getragene Zukunft überführt werden. 
